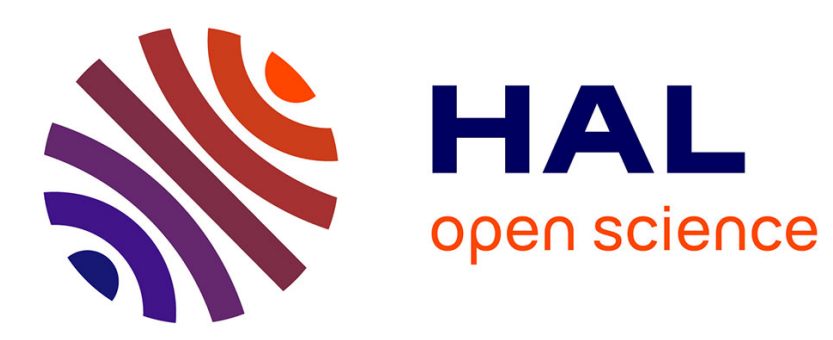

\title{
Identifiability problem for recovering the mortality rate in an age-structured population dynamics model
}

\author{
Antoine Perasso, Ulrich Razafison
}

\section{To cite this version:}

Antoine Perasso, Ulrich Razafison. Identifiability problem for recovering the mortality rate in an age-structured population dynamics model. 2014. hal-00995676

\author{
HAL Id: hal-00995676 \\ https://hal.science/hal-00995676 \\ Preprint submitted on 23 May 2014
}

HAL is a multi-disciplinary open access archive for the deposit and dissemination of scientific research documents, whether they are published or not. The documents may come from teaching and research institutions in France or abroad, or from public or private research centers.
L'archive ouverte pluridisciplinaire HAL, est destinée au dépôt et à la diffusion de documents scientifiques de niveau recherche, publiés ou non, émanant des établissements d'enseignement et de recherche français ou étrangers, des laboratoires publics ou privés. 


\title{
Identifiability problem for recovering the mortality rate
}

\section{in an age-structured population dynamics model}

\author{
Antoine Perasso $^{1}$, Ulrich Razafison ${ }^{2}$ \\ ${ }^{1}$ UMR6249 Chrono-environnement - Université de Franche-Comté \\ antoine.perasso@univ-fcomte.fr \\ ${ }^{2}$ UMR6623 Laboratoire de mathématiques de Besançon - Université de Franche-Comté \\ ulrich.razafison@univ-fcomte.fr
}

\begin{abstract}
In this article is studied the identifiability of the age-dependent mortality rate of the Von Foerster-Mc Kendrick model, from the observation of a given age group of the population. In the case where there is no renewal for the population, translated by an additional homogeneous boundary condition to the Von Foerster equation, we give a necessary and sufficient condition on the initial density that ensures the mortality rate identifiability. In the inhomogeneous case, modeled by a non local boundary condition, we make explicit a sufficicent condition for the identifiability property, and give a condition for which the identifiability problem is ill-posed. We illustrate this latter case with numercial simulations.
\end{abstract}

Mathematics Subject Classification: 35Q92, 35R30, 92D25, 93B30

Keywords: Parameter identifiability, population dynamics, age-structured model, transport PDE, non-local boundary condition

\section{Introduction}

When studying a mathematical problem that is modeling realistic and observed phenomena, an important issue is to check if the model satisfies a parameter identifiability property, i.e. whether the unknown parameters involving in the model can be uniquely determined from the observation and sometimes from the initial condition and an input of the model. This property, called parameter identifiability problem, is an inverse problem that consists to check if the parameter to output map of the model is into, initial condition and input being known. The main goal of this article is to study a parameter identifiability problem in the context of a classical age-structured population dynamics model, the McKendrick-Von Foerster model. This latter describes the evolution in time of a population structured according to the age of the individuals, leading to a problem formulated by a linear partial differential equation of transport type with a non local boundary condition. This model is classically used to describe population dynamics [13] or also in epidemiology, where the age variable represents the age of infection [4]. In this article, we wonder whether the observation of an age group of the population may recover the uniqueness of a fundamental population dynamics parameter, the mortality rate. 
To our knowledge, the identifiability analysis of the mortality rate in such a model has never been considered before. The main difficulties here are inherent of the age-dependence of the mortality rate and of the boundary condition stated by a non local loopback on the state of the system.

There is a well-established theory for the parameter identifiability of controlled and uncontrolled dynamical systems described by ordinary differential equations [2, 23, 24]. Three main approaches have been used: (i) the state isomorphism method [3, 22]; (ii) the Taylor series expansion method [18]; (iii) the algebro-differential elimination method [11, 12, 20, 19], aiming at obtaining and exploiting algebro-differential relations between the input and output of the system. In infinite dimension, identifiability results exist for fairly general classes of linear problems. Results concerning convolutive systems, which include the delay-differential equations, can be found in $[1,15,26]$. Identifiability results derived from the use of spectral theory are given in [14] for the 1-D heat and wave equations with boundary observations as well as for abstract homogeneous evolution equations with whole state observation. Results on various classes of linear models with pointwise observation where obtained using Carleman estimates, for instance for a non-stationary particle transport equation (see [10] and references therein). In the nonlinear case there are only few results dealing with transport equations using algebrodifferential approach in inifite dimension [16] or parabolic equations using either Carleman estimates [5, 8, 9] or when the observation is the state of the system [6].

This article is structured as follows. Section 2 is dedicated to the statements of the parameter identifiability problem, where an abstract formulation is performed in the linear case. Then we illustrate throughout the Malthus model how the nature of the observation may affect the identifiability problem. Section 3 contains the mortality rate identifiability analysis of the Von Foerster-McKendrick model. We introduce in section 3.1 this age-structured population model and formulate the parameter identifiability problem in Section 3.2. The identifiability analysis is split into two cases, the homogeneous case, with a null boundary condition, and the inhomogeneous case, with a non local boundary condition. Finally, we conclude with perspectives in Section 4.

\section{Statement of the identifiability problem}

\subsection{Theoretical framework}

Throughout this paper, we use the following notations,

$\mathbb{R}_{+}=[0,+\infty) ;$

$X, Y$ are Banach spaces;

$I d_{Z}$ is the identity element of the set $Z$;

$\mathscr{L}(X, Y)$, resp. $\mathscr{L}(X)$, the space of linear operators with domain $X$ and range in $Y$, resp. $X$; 
Consider the following first order linear dynamical system on $X$,

$$
\left\{\begin{array}{l}
\frac{d u(t)}{d t}=A u(t), \\
u(0)=u_{0} \in X
\end{array}\right.
$$

where $A: D(A) \subset X \rightarrow X$ is a differential operator. Given a set $P$, an unknown parameter $p \in P$ involving in system (1) and an observation linked to the state $u(t)$ of the system, we consider the model system $\mathscr{S}_{p}$, reflecting the $p$-dependence in system (1), defined by

$$
\mathscr{S}_{p}:\left\{\begin{array}{l}
\frac{d u(t ; p)}{d t}=A_{p} u(t ; p), \\
u(0)=u_{0} \in X \\
y_{p}(t)=B u(t ; p),
\end{array}\right.
$$

where $B \in \mathscr{L}(X, Y)$ is called the observation operator and function $y_{p}$ is the system output. In all that follows, we suppose the following assumptions on model system $\mathscr{S}_{p}$ :

(i) The initial condition $u_{0}$ is known.

(ii) The operator $B$ is known.

(iii) The parameter $p$ is unknown.

(iv) The operator $A_{p}$ generates a strongly continuous semigroup of operators $\left\{T_{p}(t)\right\}_{t \geq 0}$ of $\mathscr{L}(X)$.

Remark 1. From assumption (iv), it follows that system $\mathscr{S}_{p}$ has a unique solution in $X$ given by

$$
u(t)=T_{p}(t) u_{0}, \quad t \geq 0,
$$

and consequently the observation in $\mathscr{S}_{p}$ is given by $y_{p}(t)=B T_{p}(t) u_{0}$ for every $t \geq 0$. If the context is clear, the $p$-dependence of the solution $u(t ; p)$ of system $\mathscr{S}_{p}$ will be omitted.

From the given system (2), consider the system $\mathscr{S}_{\bar{p}}$ obtained by replacing $p$ by $\bar{p}$ in $\mathscr{S}_{p}$ with $\bar{p}$ satisfying assumption (iii) and $A_{\bar{p}}$ satisfying assumption (iv). For $J \subset \mathbb{R}_{+}$, the difference

$$
\epsilon(t ;(p, \bar{p}))=y_{p}(t)-y_{\bar{p}}(t), \quad t \in J,
$$

is called the output error linked to $\mathscr{S}_{p}$ and $\mathscr{S}_{\bar{p}}$ on $J$.

We now define the parameter identifiability problem.

Definition 2.1. The system $\mathscr{S}_{p}$ is said P-identifiable on $J$ if for every $p, \bar{p} \in P$ the condition $p=\bar{p}$ follows from the zero output error,

$$
\epsilon(t ;(p, \bar{p}))=0 \text { in } Y, \quad t \in J .
$$


When the equality (4) fails, we say that the parameter identifiability problem is ill-posed on $(P, J)$, or simply ill-posed on $J$ if the set $P$ is certain.

The parameter identifiability problem as stated above consists in studying if the parameter to output map of the problem is into. This study induces two equalities to work with, in one hand an equality between parameters and in other hand between the system outputs. These latter equalities require to determine the suitable functional spaces in which they are satisfied. In particular, one can check that equality (4) is satisfied in the Banach space $Y$ which is linked to the space $X$ through the relation $y=B u$. As a consequence, performing a mathematical analysis of the well posedness of the abstract Cauchy problem is a prerequisite in the study of the parameter identifiability problem.

\subsection{A short example: mortality rate identifiability in the Malthus model}

In this section is studied an identifiability problem related to the mortality rate in the classical population dynamics model of Malthus. We recall that this model is given by the following ODE system,

$$
\left\{\begin{array}{l}
\frac{d u}{d t}(t)=\beta u(t)-\mu u(t), \\
u(0)=u_{0} \in \mathbb{R}_{+},
\end{array}\right.
$$

where the quantity $u(t)$ denotes the population at time $t \geq 0, \mu \geq 0$ is the mortality rate and $\beta \geq 0$ the birth rate. We refer to [13] for the historic and a description of this model. The identifiability problem we are interested in consists in the determination of the uniqueness of parameters $\beta$ and $\mu$ together from the observation either of the whole population or of the death flow.

To achieve that goal, one can check that the notations of Section 2 rewrite the Banach space $X=\mathbb{R}$, the unknown parameter $p=\left(\begin{array}{c}\beta \\ \mu\end{array}\right) \in P=\left(\mathbb{R}_{+}^{*}\right)^{2}$ and the differential operator with domain $\mathbb{R}$ as $A_{p}=(\beta-\mu) I d_{\mathbb{R}}$. Finally, the model system $\mathscr{S}_{p}$ is linked to (5) with a given output $y_{p}$ and an observation operator $B \in \mathscr{L}(\mathbb{R})$ that are described below.

It is clear that the identifiability of the model system $\mathscr{S}_{p}$ depends on the nature of the observation. A biological intuition should make us believe that the observation of the whole population $u(t)$ at any time gives a lot of information on the parametrization of the model. The following proposition shows the opposite.

Theorem 2.2. Suppose that the operator $B: \mathbb{R} \rightarrow \mathbb{R}$ is given by

1. $B=\mu . I d_{\mathbb{R}}$, then the model system $\mathscr{S}_{p}$ is $P$-identifiable on every $J \subset \mathbb{R}_{+}$.

2. $B=I d_{\mathbb{R}}$, then the parameter identifiability problem is ill-posed on $\mathbb{R}_{+}$;

Proof. To prove the point 2 of the theorem, we have to exhibit (at least) two different parameters $p, \bar{p} \in P$ such that $\epsilon(t ;(p, \bar{p}))$ is zero for every $t \geq 0$. The integration of System (5) implies that the operator $\left(A_{p}, \mathbb{R}\right)$ is generator of the semigroup $\left\{T_{p}(t)\right\}_{t \geq 0}$ on $\mathbb{R}$ defined by

$$
T_{p}(t)=e^{(\beta-\mu) t}, \quad t \geq 0 .
$$


Then for fixed $(\beta, \mu) \in P$, the family of parameters $\left\{p_{\lambda}\right\}$ given by

$$
p_{\lambda}=\left(\begin{array}{c}
\lambda \beta \\
(\lambda-1) \beta+\mu
\end{array}\right)
$$

satisfies for every $\lambda, \lambda_{2}>\max \left(0, \frac{\beta-\mu}{\beta}\right)$,

$$
\begin{gathered}
p_{\lambda} \in P, \\
\epsilon\left(t ;\left(p_{\lambda}, p_{\lambda_{2}}\right)\right)=0, \quad t \geq 0 .
\end{gathered}
$$

As a consequence, the parameter identifiability problem is ill-posed on $\mathbb{R}_{+}$.

We now prove the point 1 of Theorem 2.2. A direct consequence of the assumption (i) about the initial data combined with the zero output error valued at $t=0$ implies that $\mu=\bar{\mu}$. Then the semigroup expression given in (6) implies the identifiability on every observation period $J \subset \mathbb{R}_{+}$.

For the observation operator $B=I d_{\mathbb{R}}$, the output is the density $u(t)$ meaning that the observation consists of the total population whereas for $B=\mu I d_{\mathbb{R}}$, the observation is the death flow. It is clear that one could either observe the birth flow, given by $B=\beta I d_{\mathbb{R}}$, and the result of Theorem 2.2.1 would still hold. Theorem 2.2 states that the knowledge of the whole population at any time is not a sufficient contribution to recover the uniqueness of parameters $\mu$ and $\beta$. On the contrary, the observation of the death flow, respectively of the birth flow, is sufficient. To conclude, Theorem 2.2 shows that the observation of a part of the population can be more useful to determine some parameters than the observation of the entirety.

Remark 2. One can check that the knowledge of $u_{0}$, stated by assumption (i) in Section 2, is necessary to conclude the identifiability result. In other word, the model system is identifiable under the condition that the state of the system has to be known for at least one time, what will be supposed in all that follows.

\section{Mortality rate identifiability in an age-structured population dy- namics model}

We start with several notations of functional spaces that will be used in all that follows.

$L^{1}\left(\mathbb{R}_{+}\right)$is the set of Lebesgue integrable functions on $\mathbb{R}_{+}$;

$L_{l o c}^{1}\left(\mathbb{R}_{+}\right)$is the set of locally Lebesgue integrable functions on $\mathbb{R}_{+}$;

$L^{\infty}\left(\mathbb{R}_{+}\right)$is the set of Lebesgue measurable functions on $\mathbb{R}_{+}$which are essentially bounded;

$W^{1,1}\left(\mathbb{R}_{+}\right)=\left\{\varphi \in L^{1}\left(\mathbb{R}_{+}\right), \varphi^{\prime} \in L^{1}\left(\mathbb{R}_{+}\right)\right\} ;$

and for any functional space $Z$ of real valued functions, the subset $Z_{+} \subset Z$ will denote the cone of non negative functions of $Z$. 


\subsection{An age structured population dynamics model}

We consider a population density $u(t, a)$ at time $t \geq 0$ and age $a \in\left[0, a_{\dagger}\right)$ where $a_{\dagger} \in(0,+\infty]$ is the upper bound of lifespan. Such a population is described by the following classical problem called Von Foerster-Mck Kendrick problem [13, 17],

$$
\left\{\begin{array}{l}
\frac{\partial u(t, a)}{\partial t}+\frac{\partial u(t, a)}{\partial a}=-\mu(a) u(t, a), \\
u(t, 0)=\int_{0}^{a_{\dagger}} b(a) u(t, a) d a \\
u(0)=u_{0}
\end{array}\right.
$$

with the assumptions

- $\mu \in L_{l o c,+}^{1}\left(0, a_{\dagger}\right)$ and $\int_{0}^{a_{\dagger}} \mu(a) d a=+\infty$;

- $b \in L_{+}^{\infty}\left(0, a_{+}\right)$;

- $u_{0} \in W_{+}^{1,1}\left(0, a_{+}\right)$.

In this model, the total number of individuals at time $t$ with age $a$ between $a_{1}$ and $a_{2}$ is

$$
\int_{a_{1}}^{a_{2}} u(t, a) d a
$$

Function $\mu$ represents an age-dependent mortality rate of the population. Assumptions made on $\mu$ mean that mortality may occur at any age and all the individuals leave the population before a finite age. As a consequence, the following quantity

$$
\pi(a)=\exp \left(-\int_{0}^{a} \mu(s) d s\right)
$$

which satisfies $\pi(0)=1$ and $\pi\left(a_{\dagger}\right)=0$, represents the probability for an individual to survive at age $a \geq 0$. Since $\mu \in L_{l o c}^{1}\left(0, a_{\dagger}\right)$, the probablity $\pi$ is clearly a continuous function on $\left[0, a_{\dagger}\right)$.

The non local boundary condition in (7), called renewal of the population, induces the birth rate $b$ which gives the repartition of birth for individuals with age $a \in\left(0, a_{\dagger}\right)$. When $b$ is zero in $L^{\infty}\left(0, a_{\dagger}\right)$, there is no renewal of the population. For more details concerning the biological significance of the assumptions of the model, we refer to [7, 25].

We now make clear the functional framework to study the abstract Cauchy problem linked to (7). To this goal, let us denote in all that follows $X=L^{1}\left(0, a_{\dagger}\right)$ and for $b \in L_{+}^{\infty}\left(0, a_{\dagger}\right)$ let $A^{b}$ : $D\left(A^{b}\right) \subset X \rightarrow X$ be the differential operator defined by

$$
\begin{gathered}
D\left(A^{b}\right)=\left\{\varphi \in W^{1,1}\left(0, a_{\dagger}\right), \varphi(0)=\int_{0}^{a_{\dagger}} b(a) \varphi(a) d a\right\}, \\
A^{b}: \varphi \mapsto-\varphi^{\prime}-\mu \varphi .
\end{gathered}
$$

The abstract Cauchy Problem related to (7) is then

$$
\left\{\begin{array}{l}
\frac{d u(t)}{d t}=A^{b} u(t), \\
u(0)=u_{0} \in W^{1,1}\left(0, a_{\dagger}\right) .
\end{array}\right.
$$

The following theorem is a classical result, see for instance [21, 25], 
Theorem 3.1. $\left(A^{b}, D\left(A^{b}\right)\right)$ is an infinitesimal generator of a strongly continuous semigroup $\left\{T^{b}(t)\right\}_{t \geq 0}$ on $X$.

Theorem 3.1 has several consequences in the study of the Cauchy Problem (9), such as existence and uniqueness of a non negative mild solution $u \in C\left(\mathbb{R}_{+}, X\right)$ of the problem, such that $(t, a) \mapsto u(t, a)$ is continuous outside the maniflod $a-t=0$. Moreover, this solution is defined in a strong sense in case where $u_{0} \in D\left(A^{b}\right)$.

\subsection{The mortality rate identifiability problem}

In this section is stated an identifiability problem related to system (9). For convenience, we suppose that $a_{\dagger}=+\infty$. It is easy (but tedious) to prove that all the identifiability results of Subsection 3.3 and 3.4 can be extended for a finite value of $a_{\dagger}$.

The parameter identifiability problem is the following: one wonders whether the observation of an age group of the population helps to recover the uniqueness of the mortality rate $\mu$ on the age domain $\mathbb{R}_{+}$, or at least on some subsets of the age domain. To study this parameter identifiability problem, we make explicit the notations of Section 2 in the context of the age-structured model (7), then we perform an abstract formulation of the identifiability problem.

In all that follows we denote, according to notations of Section 2, the set of unknown parameters

$$
P=L_{l o c,+}^{1}\left(\mathbb{R}_{+}\right)
$$

the observation operator $B \in \mathscr{L}(X)$ defined for $0 \leq a_{\star}<a^{\star} \leq+\infty$ by

$$
B: X \rightarrow X, \quad \varphi \mapsto \varphi \chi_{\left(a_{\star}, a^{\star}\right)}
$$

and the observation period

$$
J=[0, T], \quad T>0 .
$$

As stated by the notations above, the aim of the identifiability problem is to recover the uniqueness of the mortality rate $\mu \in P$ from the observation of the population density of the age group $\left(a_{\star}, a^{\star}\right) \subset \mathbb{R}_{+}$. Given $\mu, \bar{\mu} \in P$ the dependence on parameter $\mu$ of any variable $\Phi$ is denoted $\Phi_{\mu}$, and the output error related to $\mu, \bar{\mu}$ is denoted $\epsilon(t ;(\mu, \bar{\mu}))$, accordingly to Section 2 . Finally, $\mathscr{S}_{\mu}$ is the model system reflecting the dependence on the unknown parameter $\mu$, as sated in equation (2).

\section{Remark 3.}

1. The observation operator is defined from $X$ to $X$. This is a particular case where the functional space of the output is $Y=X$.

2. We can check that the divergence of the integral $\int_{0}^{+\infty} \mu=+\infty$ is not included in the definition of the set of parameters $P$. Indeed, this assumption has to be considered for its biological convenience more than as a technical need to the identifiability results.

Even if the identifiability problem is ill-posed on a time interval $J$, meaning the non uniqueness of parameter $\mu$ in $X$, it may be possible to recover the uniqueness of an unknown parameter on some subsets of the age domain $\mathbb{R}_{+}$. To take this case under consideration, we now 
extend the definition of the parameter identifiability.

Let $K \subset \mathbb{R}_{+}$be a compact subset such that $|K|>0$ where $|\cdot|$ denotes the Lebesgue measure. Then the application $\|\cdot\|_{L^{1}(K)}$ defines a semi-norm on $L_{l o c}^{1}\left(\mathbb{R}_{+}\right)$. Consider now the quotient projection

$$
\theta_{K}: L_{l o c}^{1}\left(\mathbb{R}_{+}\right) \rightarrow L_{l o c}^{1}\left(\mathbb{R}_{+}\right) / \operatorname{ker}\|\cdot\|_{L^{1}(K)}
$$

This projection has the following sense: for a function $f \in L_{l o c}^{1}\left(\mathbb{R}_{+}\right)$, the equivalence class is given by $\theta_{K}(f)=\left\{g \in L_{l o c}^{1}\left(\mathbb{R}_{+}\right), f(a)=g(a)\right.$ f.a.e $\left.a \in K\right\}$. From here, we have the following definition,

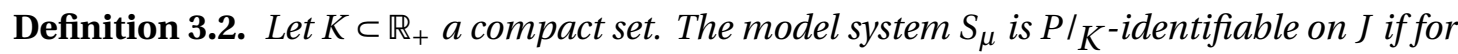
every $\mu, \bar{\mu} \in P$ the equality $\theta_{K}(\mu)=\theta_{K}(\bar{\mu})$ follows from the zero output error

$$
\epsilon(t ;(\mu, \bar{\mu}))=0, \quad t \in J .
$$

The latter definition, which allows to recover the uniqueness of the restriction of the unknown parameter $\mu$ on some compact subsets of $\mathbb{R}_{+}$, will be frequently used in the sequel.

Taking into account Problem (7) we consider in all that follows the notations

$$
\begin{gathered}
\mathscr{A}=\left\{a \in \mathbb{R}_{+}, u_{0}(a)>0\right\}, \quad \underline{a}=\inf \mathscr{A}, \\
K^{\star}=\left[0, a^{\star}\right] \text { and } K_{\star}=\left[0, a_{\star}\right], \\
\mathscr{A}^{\star}=\mathscr{A} \cap K^{\star} \text { and } \mathscr{A}_{\star}=\mathscr{A} \cap K_{\star} .
\end{gathered}
$$

The study of the mortality rate identifiability problem is split into two cases, the homogeneous case, that describes a population without renewal, and the inhomogeneous case, that is a population subject to renewal.

\subsection{Identifiability results in the homogeneous case}

We suppose in this case that the population is not subject to renewal, meaning that the boundary condition in (7) is homogeneous, translated by $b=0$ in $L^{\infty}\left(\mathbb{R}_{+}\right)$. In that case, a necessary and sufficient condition on the initial density is given to ensure the uniqueness of the mortality rate $\mu$ on $K^{\star}$, the largest reachable compact subset of the age domain $\mathbb{R}_{+}$.

Lemma 3.3. Let $T>a_{\star}$ be arbitrary but fixed and $K=\overline{\mathscr{A}^{\star}} \cup\left[a_{\star}, a^{\star}\right]$. If $\underline{a}=0$, then the model

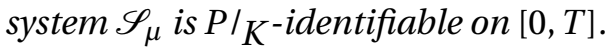

Proof. Using the characteristics of the transport equation in (7), we check that the expression of the semigroup $\left\{T_{\mu}^{0}(t)\right\}_{t \geq 0}$ generated by $\left(A^{0}, D\left(A^{0}\right)\right)$ is given for every $\varphi \in L^{1}\left(\mathbb{R}_{+}\right)$by

$$
T_{\mu}^{0}(t) \varphi(a)= \begin{cases}\pi(a)(\pi(a-t))^{-1} \varphi(a-t) & \text { for } t \leq a, \\ 0 & \text { for } 0 \leq a \leq t .\end{cases}
$$

A consequence is the following alternative expression for the output,

$$
y_{\mu}(t)=B T_{\mu}^{0}(t) u_{0}
$$


inducing that the output in model system $S_{p}$ satisfies for $(t, a) \in[0, T] \times \mathbb{R}_{+}$

$$
y_{\mu}(t, a+t)=u_{0}(a) \pi(a+t)(\pi(a))^{-1} \chi_{\left(a_{\star}, a^{\star}\right)}(a+t) .
$$

Consider now two parameters $\mu, \bar{\mu} \in P$ such that for $t \in[0, T]$ the following equality holds in $L^{1}\left(\mathbb{R}_{+}\right)$,

$$
\epsilon(t ;(\mu, \bar{\mu}))=0 .
$$

Using the expression (11) of the output we then have for every $(t, a) \in[0, T] \times \mathbb{R}_{+}$,

$$
u_{0}(a)\left(\pi(a+t)(\pi(a))^{-1}-\bar{\pi}(a+t)(\bar{\pi}(a))^{-1}\right) \chi_{\left(a_{\star}, a^{\star}\right)}(a+t)=0
$$

where $\pi$, resp. $\bar{\pi}$, is a notation used for convenience instead of $\pi_{\mu}$, resp. $\pi_{\bar{\mu}}$.

Performing the change of variables $(t, a) \mapsto(a+t, a)=(\xi, a)$ in the latter equation yields on $\left\{(\xi, a) \in\left(a_{\star}, a^{\star}\right) \times \mathscr{A}, \max (0, \xi-T) \leq a \leq \xi\right\}$,

$$
\pi(a)(\bar{\pi}(a))^{-1}=\pi(\xi)(\bar{\pi}(\xi))^{-1} .
$$

Since $T>a_{\star}$ and $\pi, \bar{\pi}$ are continuous functions, equation (13) implies that there exists a constant $c(\underline{a})$ independent of $a$ and $\xi$ such that

$$
\begin{aligned}
& \pi(a)(\bar{\pi}(a))^{-1}=c(\underline{a}), \quad \forall a \in \overline{\mathscr{A}^{\star}}, \\
& \pi(\xi)(\bar{\pi}(\xi))^{-1}=c(\underline{a}), \quad \forall \xi \in\left[a_{\star}, a^{\star}\right] .
\end{aligned}
$$

If $\underline{a}=0$, then necessarily $c(0)=1$ since $\pi, \bar{\pi}$ are probabilities, which implies

$$
\pi(a)=\bar{\pi}(a), \quad \forall a \in K,
$$



Theorem 3.4. Let $T>a_{\star}$ be arbitrary but fixed. Then the model system $\mathscr{S}_{\mu}$ is $P / K^{\star}$-identifiable on $[0, T]$ if and only if $\mathscr{A}_{\star}$ is a dense subset of $K_{\star}$.

Proof. If $\mathscr{A}_{\star}$ is a dense subset of $K_{\star}$, then $\underline{a}=0$ and $\overline{\mathscr{A}^{\star}} \cup\left[a_{\star}, a^{\star}\right]=K^{\star}$, so the sufficient condition is a direct consequence of Proposition 3.3.

We now aim at proving that the condition is necessary. To achieve that goal, we build two different parameters $\mu, \bar{\mu} \in P / K^{\star}$ such that the zero output error stands in $Y$. If $\mathscr{A}_{\star}$ is not dense in $K_{\star}$, there exists a non empty interval $U=\left(a_{-}, a^{+}\right) \subset K_{\star} \backslash \mathscr{A}_{\star}$. Let us consider $\mu$ and $\bar{\mu} \in P$ such that

$$
\begin{array}{ll}
\text { (i) } & \|\mu-\bar{\mu}\|_{L^{1}(U)}>0, \\
\text { (ii) } & \pi(a)=\bar{\pi}(a), \quad \forall a \in \mathbb{R}_{+} \backslash U .
\end{array}
$$

where $\pi$, resp. $\bar{\pi}$, is the probability to survive defined in (8) and linked to $\mu$, resp. to $\bar{\mu}$. One can check that condition (i) clearly implies that $\mu$ and $\bar{\mu}$ are two different parameters of $P / K^{\star}$. In order to reconstruct the outputs $y_{\mu}(t)$ and $y_{\bar{\mu}}(t)$ over time, the difference yielded by (i) is offset in (ii) by imposing that the probabilities to survive $\pi$ and $\bar{\pi}$ are the same outside $U$. One can check that this also implies that $\mu(a)=\bar{\mu}(a)$ f.a.e. $a \in \mathbb{R}_{+} \backslash U$. 
For a fixed $t \geq 0$, we now reconstruct the outputs $y_{\mu}(t)$ and $y_{\bar{\mu}}(t)$ on some subsets of the age domain $\mathbb{R}_{+}$, in order to prove that the output error is 0 in $X$. Due to (10), it is obvious to check that

$$
\epsilon(t ;(\mu, \bar{\mu})) \chi_{(0, t)}=0 \text { in } X .
$$

We now prove that the zero value of the outpu is satisfied for $a \in(t,+\infty)$. Suppose that $a \geq t$, and consider the three following cases.

Case $a \in\left[t+a^{+},+\infty\right)$

Then $a-t \geq a^{+}$and $a \geq a^{+}$so (ii) implies that $\pi(a-t)=\bar{\pi}(a-t)$ and $\pi(a)=\bar{\pi}(a)$. Equation (10) then yields

$$
u(t, a ; \mu)=u(t, a ; \bar{\mu})
$$

Case $a \in\left[t+a_{-}, t+a^{+}\right]$

Then $a-t \in U \subset K_{\star} \backslash \mathscr{A}_{\star}$ so $u_{0}(a-t)=0$ and (14) still holds.

Case $a \in\left[a^{+}, t+a_{-}\right]$

This latter case holds when $t \geq a^{+}-a_{-}$. Then $a-t \leq a_{-}$and assumption (ii) implies that $\pi(a-$ $t)=\bar{\pi}(a-t)$. Moreover, since $a \geq a^{+}$we also have $\pi(a)=\bar{\pi}(a)$. So equality (14) is satisfied.

To conclude the several cases, equality (14) is satisfied for $a \in[t,+\infty) \cap\left[\min \left(a^{+}, t+a_{-}\right),+\infty\right)$. Finally, since $U \subset K_{\star}$, then either the observed age period satisfies $\left(a_{\star}, a^{\star}\right) \cap[t,+\infty) \subset\left[\min \left(a^{+}, t+\right.\right.$ $\left.\left.a_{-}\right),+\infty\right)$ or $\left(a_{\star}, a^{\star}\right) \cap[t,+\infty)=\varnothing$ so one gets

$$
\epsilon(t ;(\mu, \bar{\mu})) \chi_{(t,+\infty)}=0 \text { in } X,
$$

which ends the proof.

\section{Remark 4.}

1. The result of Theorem 3.4 can be easily extended to the case where $a^{\star}=+\infty$, meaning that every individual of the population with age larger than $a_{\star}$ is observed. In this particular case of observation, the model system is $P$-identifiable on $[0, T]$ if and only if $\mathscr{A}$ is dense in $\mathbb{R}_{+}$.

2. In order to get the mortality rate identifiability, one could believe that it is necessary to observe the population on an horizon time $T$ larger than $a^{\star}$ the maximum age of observation. Theorem 3.4 shows that $T>a_{\star}$ is sufficient.

\subsection{Identifiability results in the inhomogeneous case}

We now suppose that the population is subject to reproduction, implying an inhomogeneous boundary condition in (7) since $b$ is not zero in $L^{\infty}\left(\mathbb{R}_{+}\right)$. This section shows that the boundary condition yields a fundamental perturbation in the study of the mortality rate identifiability problem. Indeed, whereas the sufficient condition on the initial density $u_{0}$ stated in Theorem 3.4 still holds to prove the identifiability result, a necessary condition on the initial density and on the boundary condition via the birth function $b$ is stated in Theorem 3.5. Moreover, to prove that this necessary condition is not a sufficient one, we exhibit a subclass of parameters of $P$ that satisfy an identifiability property whereas $u_{0}$ and $b$ are such that the necessary condition does not hold. This is done in Proposition 3.6. 


\section{Extension of Theorem 3.4 in the inhomogeneous case}

\section{Theorem 3.5.}

1. Let $T>a_{\star}$ be arbitrary but fixed. If $\mathscr{A}_{\star}$ is dense in $K_{\star}$ then the model system $\mathscr{S}_{\mu}$ is $P / K^{\star}-$ identifiable on $[0, T]$.

2. If there exists an open set $U \subset K_{\star} \backslash \mathscr{A}_{\star}$ such that $b(a)=0$ f.a.e $a \in U$, then the parameter identifiability problem is ill-posed on $[0, T]$ for any $T \geq 0$.

Proof. Contrary to homogeneous boundary condition case, where equation (10) gives an explicit expression of the semigroup $\left\{T^{0}(t)\right\}_{t \geq 0}$, it is not possible to make explicit such an expression for $\left\{T^{b}(t)\right\}_{t \geq 0}$ when $b$ is not zero in $L^{\infty}\left(\mathbb{R}_{+}\right)$, which is due to the non local loopback boundary condition in (7). However, a change of variables using the characteristics of the transport equation proves that the state of the system $u(t, a)$ satisfies the following implicit formulation

$$
u(t, a)=\left\{\begin{array}{l}
\pi(a)(\pi(a-t))^{-1} u_{0}(a-t) \quad \text { for } t \leq a, \\
\pi(a) u(t-a, 0) \quad \text { for } 0 \leq a \leq t,
\end{array}\right.
$$

that will be used in the following. From this expression, one can check that the proof of the point 1. of Theorem 3.5 holds by the same arguments that ones developed to prove the sufficient condition in Theorem 3.4.

So we now focus on the proof of the point 2 of the theorem. Without lack of generality, one can suppose that $U=\left(a_{-}, a^{+}\right)$an open interval and consider two parameters $\mu, \bar{\mu} \in P$ that satisfy the assumptions (i)-(ii) as in the proof of Theorem 3.4. To prove that the output error is zero, the main difficulty is to get the zero value for $a \in(0, t)$. Indeed, (15) implies that it is necessary to reconstruct the boundary conditions $u(t, 0 ; \mu)$ and $u(t, 0 ; \bar{\mu})$ to reach that goal.

Equation (15) obviously implies, as in the proof of Theorem 3.4, the following zero value of the output error,

$$
\epsilon(t ;(\mu, \bar{\mu})) \chi_{(t,+\infty)}=0 \text { in } X .
$$

Moreover, equation (15) implies that the difference between boundary conditions in (7) rewrites for every $t \geq 0$,

$$
\begin{array}{r}
u(t, 0 ; \mu)-u(t, 0, \bar{\mu})=\int_{0}^{t} b(a)(u(t-a, 0 ; \mu) \pi(a)-u(t-a, 0 ; \bar{\mu}) \bar{\pi}(a)) d a \\
+\int_{t}^{+\infty} b(a)(u(t, a, \mu)-u(t, a ; \bar{\mu})) d a .
\end{array}
$$

Let us prove that the latter integral in the equation above is zero. To achieve that goal, one can check that equality (14) can be extended on $[t,+\infty) \cap\left[0, a_{-}\right]$. Indeed, in case where $a \in\left[0, a_{-}\right]$ one gets $a-t \leq a \leq a_{-}$and assumption (ii) implies $\pi(a-t)=\bar{\pi}(a-t)$ and $\pi(a)=\bar{\pi}(a)$, so we finally get

$$
u(t, a ; \mu)=u(t, a ; \bar{\mu}), \quad \forall a \in[t,+\infty) \cap E_{t},
$$

where $E_{t}=\left[0, a_{-}\right] \cup\left[\min \left(a^{+}, t+a_{-}\right),+\infty\right)$. Then (17) yields

$$
\int_{t}^{+\infty} b(a)(u(t, a, \mu)-u(t, a ; \bar{\mu})) \chi_{E_{t}}(a) d a=0, \quad \forall t \geq 0 .
$$


Moreover, from the definition of $E_{t}$ one deduces that $\bigcup_{t \geq 0}\left(\mathbb{R}_{+} \backslash E_{t}\right) \subset U$, and since $b(a)=0$ f.a.e. $a \in$ $U$, one gets

$$
\int_{t}^{+\infty} b(a)(u(t, a, \mu)-u(t, a ; \bar{\mu})) \chi_{\mathbb{R}_{+} \backslash E_{t}}(a) d a=0, \quad \forall t \geq 0 .
$$

Consequently, the difference between boundary conditions satisfies

$$
u(t, 0 ; \mu)-u(t, 0, \bar{\mu})=\int_{0}^{t} b(a)(u(t-a, 0 ; \mu) \pi(a)-u(t-a, 0 ; \bar{\mu}) \bar{\pi}(a)) d a .
$$

From here, since $b(a)=0$ f.a.e. $a \in U$ and $\pi(a)=\bar{\pi}(a)$ for every $a \in \mathbb{R}_{+} \backslash U$ consequently to assumption (ii), a change of variables implies that the difference $f(t)=u(t, 0 ; \mu)-u(t, 0, \bar{\mu})$ is a solution of the following Volterra integral equation

$$
f(t)=\int_{0}^{t} b(t-a) \pi(t-a) f(a) d a, \quad \forall t \geq 0 .
$$

Since $b \in L^{\infty}\left(\mathbb{R}_{+}\right)$and $\pi$ is a probability, such a function $f$ satisfies

$$
|f(t)| \leq\|b\|_{L^{\infty}} \int_{0}^{t} f(a) d a, \quad \forall t \geq 0,
$$

and a standard Gronwall argument implies

$$
|f(t)| \leq f(0) e^{t\|b\|_{L^{\infty}}}, \quad \forall t \geq 0 .
$$

Moreover, the assumption (i) stated in Section 2 on the initial condition yields

$$
f(0)=\int_{0}^{+\infty} b(a)\left(u_{0}(a ; \mu)-u_{0}(a ; \bar{\mu})\right) d a=0,
$$

and (18) finally gives

$$
u(t, 0 ; \mu)-u(t, 0, \bar{\mu})=0, \quad \forall t \geq 0 .
$$

From here, since $U \subset K_{\star}$ the infimum $a_{\star}$ of the age range of observation satisfies $a_{\star} \geq a^{+}$and one gets from assumption (ii) (proof of Th.3.4), $\pi(a)=\bar{\pi}(a)$ for $a \in\left[a_{\star}, a^{\star}\right]$, so (19) combined with (15) imply

$$
\epsilon(t ;(\mu, \bar{\mu})) \chi_{(0, t)}=0 \text { in } X .
$$

Equations (16) and (20) together end the proof.

Illustration of the ill-posed case A consequence of Theorem 3.5.2 is that for a population that is composed of juveniles and adults that is described by the age-structured model (7), it is necessary to start the observation when birth occur to get the identifiability of mortality rate $\mu$. Let us illustrate that case by considering a population with the following characteristics:

(i) Population Density: $u(t, a)$ with $t \geq 0$ and $a \in[0,1)$;

(ii) Population with juveniles: $\exists a_{\star} \in(0,1)$ such that $b(a)=0$ f.a.e. $a \in\left(0, a_{\star}\right)$; 




Figure 1: Mortality rate $\mu$ and perturbations $\bar{\mu}_{\alpha_{i}}$ for $i \in\{1,2,3\}$.

(iii) Observation of adults: output $y_{\mu}=u(t, \cdot) \chi_{\left(a_{\star}, 1\right)}$;

(iv) No birth at the begining of the observation: there exists $\epsilon \in\left(0, a_{\star}\right]$ such that $u_{0}(a)=0$ for every $a \in[0, \epsilon]$.

Hypotheses of Theorem 3.5.2 are satisfied with $K:=K_{\star}=K^{\star}=\left[0, a_{\star}\right], \mathscr{A}_{\star}=\varnothing$ and $U=\left(0, a_{\star}\right) \subset$ $K \backslash \mathscr{A}_{\star}$. This latter theorem then states that there exists different mortality rates of $P$ that lead to the same observations, or in other words that it is possible to find $\mu, \bar{\mu} \in P$ such that $\theta_{K}(\mu) \neq$ $\theta_{K}(\bar{\mu})$ but $y_{\mu}=y_{\bar{\mu}}$. We therefore aim at building such different mortality rates leading to the same observation.

Consider the mortality rate $\mu \in P$ given by

$$
\mu(x)=c\left(\frac{x}{a_{\star}} \chi_{\left[0, a_{\star}\right]}+\frac{1-a_{\star}}{1-x} \chi_{\left[a_{\star}, 1\right)}\right), \quad c>0
$$

To illustrate the ill-posed case, we build a parameter $\bar{\mu} \in P$ by performing some perturbations of $\mu$ on $K=\left[0, a_{\star}\right]$ such that $\theta_{K}(\mu) \neq \theta_{K}(\bar{\mu})$ while $y_{\mu}(t, a)=y_{\bar{\mu}}(t, a)$. In other word, we seek to maximize $\bar{\mu} \in P \mapsto\|\mu-\bar{\mu}\|_{L^{1}(K)}$ under the constraint $\left\{\pi\left(a_{\star}\right)=\bar{\pi}\left(a_{\star}\right)\right\}$. To reach that goal, let us choose mortality rates as an affine perturbation on $K$ of $\mu$ given for any $\alpha \in\left(0, \frac{a_{\star}}{2}\right]$ by

$$
\bar{\mu}_{\alpha}(x)=\frac{c x}{2 \alpha} \chi_{[0, \alpha]}+\frac{c}{2} \chi_{\left[\alpha, a_{\star}-\alpha\right]}+c\left(\frac{x-a_{\star}}{2 \alpha}+1\right) \chi_{\left[a_{\star}-\alpha, a_{\star}\right]}+\mu(x) \chi_{\left[a_{\star}, 1\right)} .
$$

One can easily check that $\theta_{K}\left(\bar{\mu}_{\alpha}\right)=\theta_{K}(\mu)$ when $\alpha=\frac{a_{\star}}{2}$ and $\theta_{K}\left(\bar{\mu}_{\alpha}\right) \neq \theta_{K}(\mu)$ else. Figure 1 shows the mortality rate $\mu$ and some perturbations $\bar{\mu}_{\alpha}$, where the numerical values are given below.

The simulations are made using an upwind scheme based on finite volume method with the following numerical values:

- $T=3, a_{\star}=0.4, \epsilon=0.4, c=4, \alpha_{i}=\frac{a_{\star}}{3^{i}}, i \in \mathbb{N}$ 


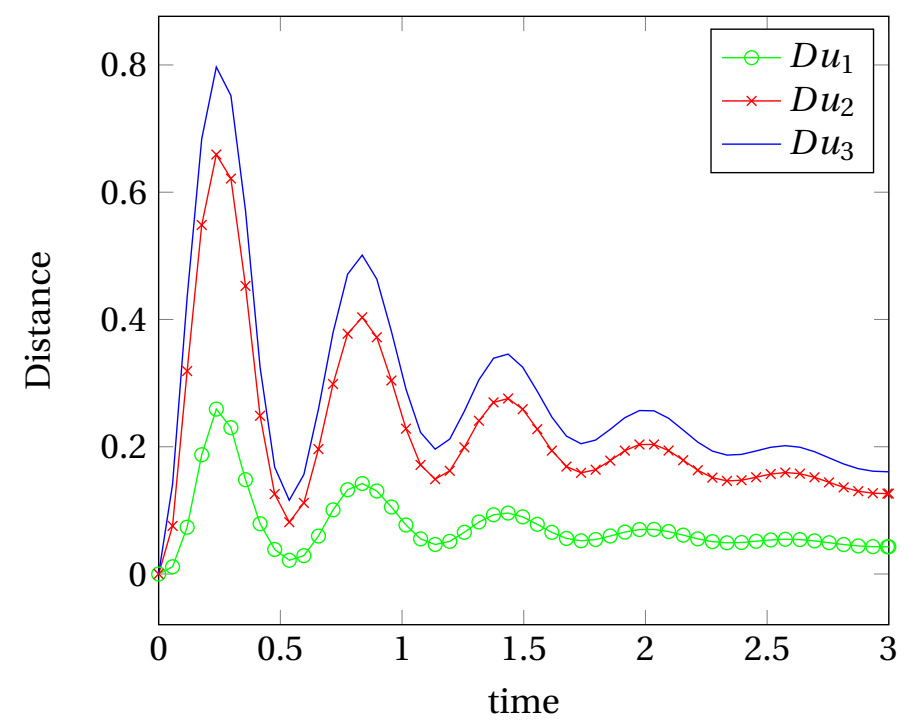

Figure 2: Distance $D u_{i}=\left\|u(t ; \mu)-u\left(t ; \bar{\mu}_{\alpha_{i}}\right)\right\|_{L^{1}(0,1)}$ for $i \in\{1,2,3\}$.

- $u_{0}(a)=\left(1-\chi_{\left[0, a_{\star}\right]}\right)+100\left(a-a_{\star}\right) \chi_{\left[a_{\star}, a_{\star}+0.1\right]}+10 \chi_{\left[a_{\star}+0.1,1\right]}$

- $b(a)=\left(1-\chi_{\left[0, a_{\star}\right]}\right)+200\left(-a^{2}+\left(1+a_{\star}\right) x-a_{\star}\right)$

As we could expect, these simulations illustrate well the ill-posed case. Indeed, whereas Figure 2 shows that the $\mathrm{L}^{1}$-norms between $u$ and $u_{i}$ are different, Figure 3 shows that, at final time $t=T$, the ouptuts are superposed and consequently nondifferentiable at any age that is observed, even if the densities $u$ and $u_{i}$ are different before the age of observation $a_{\star}=0.4$. Finally, Figure 4 shows that the superposition of the curves for ages upper $a_{\star}$ holds for any time $t \leq T$.

The sufficient condition in Theorem 3.5 is not necessary Contrary to Theorem 3.4 that states in the homogeneous case a necessary and sufficient condition dealing with the initial density to get identifiability, we can see in Theorem 3.5 that the loopback boundary condition yields a fundamental perturbation in the study of the parameter identifiability problem. Indeed, the following result shows that when dealing with a non homogeneous boundary condition, the density of $\mathscr{A}_{\star}$ in $K_{\star}$ is not a necessary condition to get identifiability on $P$.

Let us suppose that there exists an open interval $\left(a_{-}, a^{+}\right) \subset K^{\star}$ such that

$\left(a_{1}\right) \quad u_{0}(a)=0$ and $b(a)>0$ f.a.e. $a \in\left(a_{-}, a^{+}\right)$

$\left(a_{2}\right) \quad u_{0}(a)>0$ and $b(a)=0$ f.a.e. $a \in \mathbb{R}_{+} \backslash\left(a_{-}, a^{+}\right)$

$\left(a_{3}\right) a^{+} \leq 2 a_{-}$

It is easy to check that such initial density $u_{0}$ and birth rate $b$ do not satisfy the assumption of Theorem 3.5 since they do not vanish simultaneously. 


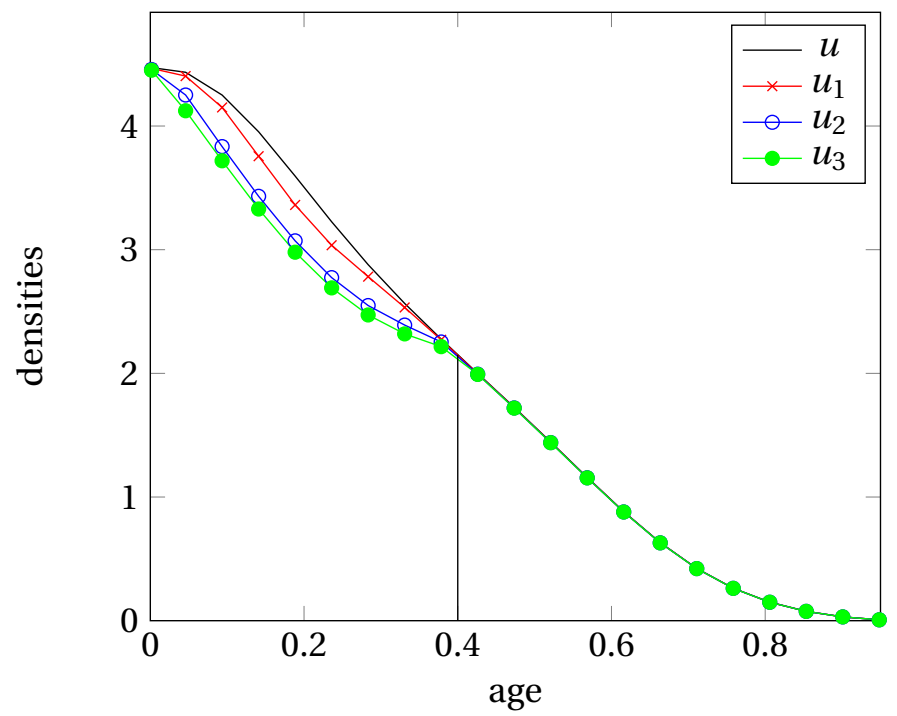

Figure 3: Age-structured densities $u=u(T ; \mu)$ and $u_{i}=u\left(T ; \bar{\mu}_{\alpha_{i}}\right), i \in\{1,2,3\}$, at final time $t=T$.

Proposition 3.6. Suppose that there exists an open interval $\left(a_{-}, a^{+}\right)$such that $\left(a_{1}\right)-\left(a_{3}\right)$ hold and let $T>a_{\star}+a_{-}$be arbitrary but fixed. Then the model system $\mathscr{S}_{\mu}$ is $P / K^{\star}$-identifiable on $[0, T]$.

Proof. Let $T>a_{\star}+a_{-}$and consider $\mu, \bar{\mu} \in P$ such that $\epsilon(t ;(\mu, \bar{\mu}))=0$ in $X$ for $t \in[0, T]$.

To prove the identifiablity result, we aim at proving that the probabilities to survive satisfy

$$
\pi(a)=\bar{\pi}(a), \quad \forall a \in\left[0, a^{\star}\right]
$$

We recall that the population density $u(t, a)$ satisfies the equality (15). Then for $a \in\left(a_{\star}, a^{\star}\right)$ and $t \leq a$ the zero value of the output error implies

$$
u_{0}(t)\left(\pi(a)(\pi(t))^{-1}-\bar{\pi}(a)(\bar{\pi}(t))^{-1}\right)=0 .
$$

From here, assumption $\left(a_{2}\right)$ gives $\pi(a)(\pi(t))^{-1}=\bar{\pi}(a)(\bar{\pi}(t))^{-1}$ on $\left\{(t, a) \in[0, T] \backslash\left(a_{-}, a^{+}\right) \times\left(a_{\star}, a^{\star}\right), a \geq\right.$ $t\}$, which is non empty since $T>a^{-}$. A direct consequence of the continuity of the probability functions $\pi, \bar{\pi}$ is then

$$
\pi(a)=\bar{\pi}(a), \quad \forall a \in\left[0, a_{-}\right] \cup\left[\min \left(a_{\star}, a^{+}\right), a^{\star}\right]
$$

If $a_{\star} \leq a_{-}$then $\pi=\bar{\pi}$ on $K^{\star}$. Suppose now that $a_{\star}>a_{-}$and let us prove that $\pi=\bar{\pi}$ on $\left[a_{-}, a^{+}\right]$. To prove this result, one can firstly check that the boundary conditions linked to $\mu$ and $\bar{\mu}$ are identically on the horizon time $\left[0, T-\min \left(a_{\star}, a^{+}\right)\right]$. Indeed the zero value of the output error and the characteristic expression (15) imply that for $a \in\left(a_{\star}, a^{\star}\right)$ and $t \geq a$,

$$
\pi(a) u(t-a, 0 ; \mu)=\bar{\pi}(a) u(t-a, 0 ; \bar{\mu}),
$$

and (22) then gives

$$
u(t, 0 ; \mu)=u(t, 0 ; \bar{\mu}), \quad \forall t \in\left[0, T-\min \left(a_{\star}, a^{+}\right)\right] .
$$




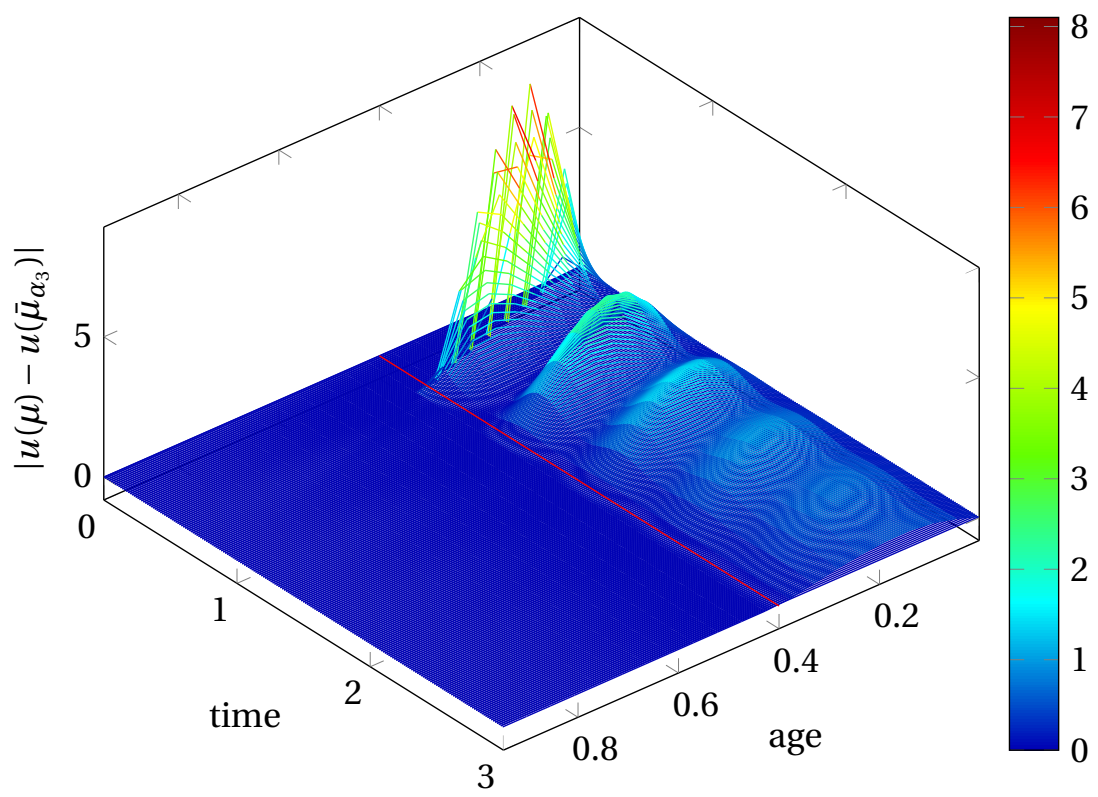

Figure 4: Difference between $u(\mu)$ and $u\left(\bar{\mu}_{\alpha_{3}}\right)$ over age and time.

From the definition of the boundary condition in Problem (7) and assumption $\left(a_{2}\right)$ on $b$, the latter equation yields

$$
\int_{a_{-}}^{a^{+}} b(a)(u(t, a ; \mu)-u(t, a ; \bar{\mu})) d a=0, \quad \forall t \in\left[0, T-\min \left(a_{\star}, a^{+}\right)\right] .
$$

Since $T-\min \left(a_{\star}, a^{+}\right) \geq a_{-}$one can consider (23) for $t \in\left[0, a_{-}\right]$. Then using the expression on characteristics (15), equation (22) and assumption $\left(a_{1}\right)$ on $u_{0}$ one gets the equality

$$
\int_{a_{-}}^{\min \left(t+a_{-}, a^{+}\right)} \frac{b(a) u_{0}(a-t)}{\pi(a-t)}(\pi(a)-\bar{\pi}(a)) d a=0, \quad \forall t \in\left[0, a_{-}\right] .
$$

Consider now the set $\mathscr{E}=\left\{a \in\left[a_{-}, a^{+}\right], \pi(x)=\bar{\pi}(x) \quad \forall x \leq a\right\}$. The set $\mathscr{E}$ is clearly non empty since $a_{-} \in \mathscr{E}$ so one can consider $a_{0}=\max \mathscr{E}$. Suppose by contradiction that $a_{0}<a^{+}$. Then there exists $0<\delta \leq a^{+}-a_{0}$ such that $\pi(a)-\bar{\pi}(a) \neq 0$ for every $a \in\left(a_{0}, a_{0}+\delta\right)$.

Consider a fixed $t \in\left(a_{0}-a_{-}, \delta+a_{0}-a_{-}\right)$. Then we have $t+a_{-} \leq a^{+}$. Moreover, from assumption $\left(a_{3}\right)$ we have $\delta+a_{0}-a_{-} \leq a_{-}$and so $t<a_{-}$. Consequently equation (24) rewrites for $t \in\left(a_{0}-\right.$ $\left.a_{-}, \delta+a_{0}-a_{-}\right)$as

$$
\int_{a_{0}}^{t+a_{-}} \frac{b(a) u_{0}(a-t)}{\pi(a-t)}(\pi(a)-\bar{\pi}(a)) d a=0 .
$$

The latter equality implies a contradiction since in one hand $\frac{b(a) u_{0}(a-t)}{\pi(a-t)}>0$ f.a.e. $a \in\left(a_{0}, t+a_{-}\right)$ and in other hand $\pi(a)-\bar{\pi}(a) \neq 0$ for every $a \in\left(a_{0}, t+a_{-}\right) \subset\left(a_{0}, a_{0}+\delta\right)$.

So $a^{+}=\max \mathscr{E}$ and $\pi=\bar{\pi}$ on $\left[a_{-}, a^{+}\right]$. Finally, the equation (22) then implies that $\mu(a)=\bar{\mu}(a)$ f.a.e $a \in K^{\star}$ which proves that the model system $\mathscr{S}_{\mu}$ is $P / K^{\star}$-identifiable on $[0, T]$. 


\section{Perspectives}

The present work, dealing with the mortality rate identifiability in an age-structured model, shows how the non local loopback boundary condition affects the study of the uniqueness of the parameter. The inverse problem is studied from the expression of the semigroup generated by the differential operator. One think that the present results could be adpated to the identifiability of functional parameters, such as growth function, in the case of size-structured models. Indeed, the latter models are described by transport equations with non constant velocities in the transport term, that may derive a semigroup formulation as in age-structured models.

\section{References}

[1] L. Belkoura. Identifiability of systems described by convolution equations. Automatica, 41(3):505-512, 2005.

[2] M. J. Chappell, K. R. Godfrey, and S. Vajda. Global identifiability of the parameters of nonlinear-systems with specified inputs: a comparison of methods. Math. Biosci., 102(1):41-73, 1990.

[3] L. Denis-Vidal and G. Joly-Blanchard. Equivalence and identification analysis of uncontrolled nonlinear dynamical systems. Automatica, 40(2):287-292, 2004.

[4] O. Diekmann and J. A. P. Heesterbeek. Mathematical Epidemiology of Infectious Diseases. Wiley Series in Mathematical and Computational Biology. John Wiley \& Sons, 1995.

[5] H. Egger, H. W. Engl, and M. V. Klibanov. Global uniqueness and hölder stability for recovering a nonlinear source term in a parabolic equation. Inverse Problems, 21(1):271-290, 2005.

[6] S. Handrock-Meyer. Identifiability of distributed parameters for a class of quasilinear differential equations. J. Inv. Ill-Posed Problems, 5:5-28, 1997.

[7] M. Iannelli. Mathematical Theory of Age-Structured Population Dynamics. Giardini editori e Stampatori, Pisa, 1995.

[8] B. Kaltenbacher and M. V. Klibanov. An inverse problem for a nonlinear parabolic equation with applications in population dynamics and magnetics. SIAM J. Math. Anal., 39(6):18631889, 2008.

[9] M. Klibanov. Global uniqueness of a multidimensional inverse nonlinear parabolic equation by a carleman estimate. Inverse Problems, 20(4):1003-1032, 2004.

[10] M. Klibanov, V. Michael, and S. E. Pamyatnykh. Global uniqueness for a coefficient inverse problem for the non-stationary transport equation via carleman estimate. J. Math. Anal. Appl., 343(1):352-365, 2008.

[11] L. Ljung and T. Glad. On global identifiability for arbitrary model parametrizations. J. Math. Anal. Appl., 30(2):265-276, 1994. 
[12] G. Margaria, E. Riccomagno, and L. White. Structural identifiability analysis of some highly structured families of statespace models using differential algebra. J. Math. Biol., 49(5):433-454, 2004.

[13] J.D. Murray. Mathematical Biology I, An introduction. Interdisciplinary applied mathematics. Springer, third edition edition, 2004.

[14] S. Nakagiri. Review of Japanese work of the last ten years on identifiability in distributed parameter systems. Inverse Problems, 9(2):143-191, 1993.

[15] Y. Orlov, L. Belkoura, J. P. Richard, and M. Dambrine. On identifiability of linear time-delay systems. IEEE Trans. Automat. Control, 47(8):1319-1324, 2002.

[16] A. Perasso, B. Laroche, Y. Chitour, and S. Touzeau. Identifiability analysis of an epidemiological model in a structured population. J. Math. Anal. Appl., 374(1):154-165, 2011.

[17] B. Perthame. Transport equations in biology. Birkhäuser, 2007.

[18] H. Pohjanpalo. System identifiability based on the power series expansion of the solution. Math. Biosci., 41(1-2):21-33, 1978.

[19] M. Saccomani. An effective automatic procedure for testing parameter identifiability of hiv/aids models. Bull. Math. Biol., 73:1734-1753, 2011.

[20] M. Saccomani, S. Audoly, and L. D’Angiò. Parameter identifiability of nonlinear systems: the role of initial conditions. Automatica, 39(4):619-632, 2003.

[21] H. R. Thieme. Mathematics in Population Biology. Princeton Series in Theoretical and Computational Biology. Princeton, 2003.

[22] S. Vajda, K. R. Godfrey, and H. Rabitz. Similarity transformation approach to identifiability analysis of nonlinear compartmental-models. Math. Biosci., 93(2):217-248, 1989.

[23] E. Walter. Identifiability of State Space Models. Springer-Verlag, 1980.

[24] E. Walter and L. Pronzato. Identification of Parametric Models. Springer, 1997.

[25] G.F. Webb. Dynamics of populations structured by internal variables. Math. Z., 189(3):319$335,1985$.

[26] J. Zhang, X. Xia, and C. H. Moog. Parameter identifiability of nonlinear systems with timedelay. IEEE Trans. Automat. Control, 51(2):371-375, 2006. 